International Journal of Biomedicine I June 2019 - Volume 9, Issue Suppl_1: Abstracts From the Second Russian International Conference "Cryo-electron microscopy 2019: achievements and prospects"

POSTER ABSTRACT PRESENTATIONS

SESSION TITLE: STRUCTURE OF VIRUSES AND CHAPERONINS

DOI: 10.21103/IJBM.9.Suppl_1.P31

\title{
Abstract P-31: Structure of Pseudomonas aeruginosa Luz-24-like Phage U47
}

Evgeny Pichkur ${ }^{1,2,3}$, Valery Novoseletsky ${ }^{4}$, Mikhail Shneider ${ }^{5}$, Anton Sedov ${ }^{5}$, Nina Sikilinda ${ }^{5}$, Konstantin Miroshnikov ${ }^{5}$, Olga Sokolova ${ }^{4}$

${ }^{1}$ National Research Center "Kurchatov Institute”, Moscow, Russia; ${ }^{2}$ Petersburg Nuclear Physics Institute-National Research Center "Kurchatov Institute", Gatchina, Russia; ${ }^{3}$ FSRC "Crystallography and Photonics”, Moscow, Russia; ${ }^{4}$ Lomonosov Moscow State University, Moscow, Russia; ${ }^{5}$ IBCh RAS, Moscow, Russia

Background: Phage U47 belongs to the Luz24-virus genus of the Podoviridae family. Due to the effective mechanism of host metabolism suppression and an ability to penetrate bacterial biofilms Luz24like Pseudomonas aeruginosa phages prospective for phage therapy applications. The relatively long tail of U47 makes it a promising model for structural studies of podoviruses. Yet, the structural information concerning adsorption complex of podoviruses, which is crucial for understanding of the infection process, is limited.

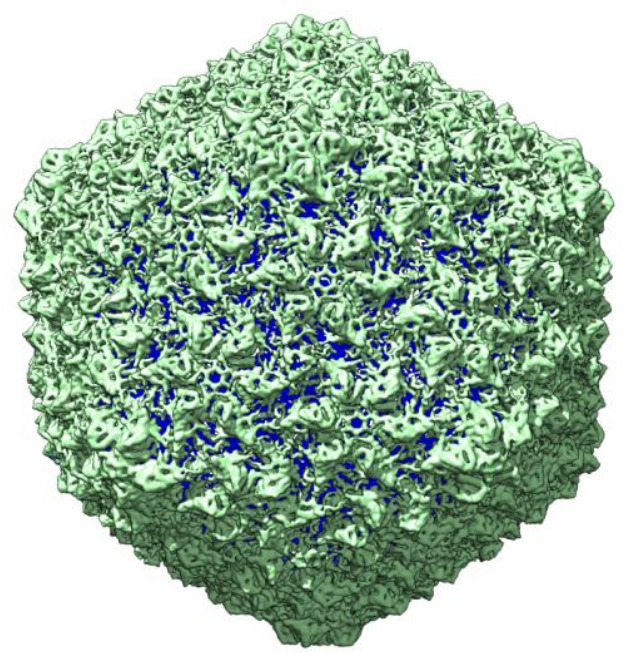

Fig. 1. Phage U47.

Methods: For the purification of the U47 phage, we used ultracentrifugation in a cesium gradient and further dialysis against buffer with low osmotic pressure. U47 solution was mixed 10:1 with $0.05 \%$ Tween 20. 3ul of the sample were applied onto the hydrophilized holey carbon support film (Quantifoil 
1.2/1.3). A total of 6500 movies were collected using cryo-TEM Krios (Thermo-Fisher, USA) at acceleration voltage $300 \mathrm{kV}$, pixel size $1.15 \AA$, flux approx. $100 \mathrm{e} / \mathrm{A}^{2} \mathrm{~s}$. Motion correction and CTF estimation was performed using Warp, particles were picked and processed in CryoSPARC and Relion 3.0. In spite of the absence of highly similar homologues molecular model of this protein was built with Modeller using HHpred for homology search. As templates surwed the structures of the major capsid proteins from bacteriophages Hk97 (pdb: 1OHG) and phi812K1-420 (pdb: 5LII). Structural model was fitted into the obtained cryo-EM density map.

Results: We have obtained the icosahedral reconstruction of the U47 phage capsid with a resolution of $4.5 \sim 6 \AA$ and the reconstruction of its tail with C6 symmetry. The elongated tail structure can be divided into 2-3 structural compartments (similar to the T7 phage; tail fibrils are visible that are attached to the distal tail protein; the tail possesses a distal spike, located parallel to the long axis of the tail, similar to that of phages P22 and SF6. Also, the globular domain is clearly visible.

Conclusion: Here, we have, for the first time, reconstructed the capsid and tail of the P.aeruginosa U47 phage and demonstrated that it possesses all the structural features of the Luz-24-like family. Further investigations should shed light to the specific adsorption complex of this phage.

Key Words: phage $\bullet$ structure $\bullet$ molecular modeling $\bullet$ cryo-EM

Sources of Funding: This work was supported by RFBR \# 19-14-00605 (cryo-EM data obtaining) and RSF \#18-41-06001 (cryo-EM data processing).

The authors would like to thank Sergey Nazarov for his helpful advice on sample preparation and data processing. The authors acknowledge the support and the use of resources of the Resource Center for Probe and Electron Microscopy at the NRC "Kurchatov Institute".

International Journal of Biomedicine. 2019;9 Suppl 1: S30-31. doi: 10.21103/IJBM.9.Suppl_1.P31 (C)2019 International Medical Research and Development Corporation 